\title{
Recall of accessible items from memory as a function of executive instructions, delay tasks, and serial position
}

\author{
BERT ZIPPEL \\ City University of New York, Hunter College, New York, New York 10021
}

\begin{abstract}
Category members or associations were accessed from memory storage for eight different stimuli, which were category labels or the most typical responses within these categories. Students produced 16 responses for each stimulus according to one of four different instructions and then, immediately or after a delay task, recalled these items. A serial position effect for the recalled items was obtained. The longer the initial latency in producing a response, especially among the earliest responses to each stimulus, the less likely the response would be recalled subsequently. The presence or absence of a delay task had no effect on recall. Recall decreased as the size of the available pool of items delimited by the instructions increased and, less effectively, as the level of complexity of the instructions increased, giving weak evidence that instructions may have some representation in short-term memory.
\end{abstract}

Murdock (1974) defined memory as dealing with the encoding, storage, and retrieval of information. Current theories suppose that there are at least three components of memory: some form of short-term sensory storage, short-term memory (STM), and long-term memory (LTM). These components may be further serviced by an executive that functions in the processing of information among these component memories (Anderson \& Bower, 1973). Research has concentrated on the way in which these component memories process information for encoding. The present research focuses on the retrieval process. Zippel (1975) demonstrated that available items accessed from storage are given in an organized fashion, in chunks of about three items, a finding consistent with the role of organization factors in encoding as documented by such authors as Johnson (1970), Mandler (1967), and Wickelgren (1964). However, it is not safe to assume that processes of accessing information from storage are the same processes that encode into storage items supplied by an experimenter.

If items accessed directly from a person's own LTM operate similarly to items initially supplied by an experimenter, then the subsequent free recall of such items should evidence the serial position effect found in typical free recall studies. However, since all items during the initial production period are by definition accessible, the asymptote of the curve should be quite high for the subsequent recall. Posner and Rossman (1965) found that the more difficult an interpolated task, the less recall from STM. Keele (1973) argued that the more difficult interpolated tasks involve more mental opera-

Reprints of this article may be obtained by writing to Bert Zippel, Box 389, City University of New York, Hunter College, 695 Park Avenue, New York, New York 10021. tions than do the simpler tasks and that those mental operations interfere with storage operations of rehearsal. If the limits of STM operate on these accessed items in recall, then the introduction of an appropriate delay task should reduce the typical increase in the curve over the last few items in recall (Glanzer, 1972).

Various executive instructions may differentially affect the subsequent recall of accessible items. Instructions must be represented somewhere in the memory process. One possibility is that instructions have some representation in STM. If that is the case, and if the total capacity of STM is limited, then complex instructions may act to displace content items from such storage. The more restrictive the executive instructions, the smaller the available population of items, as in Meyer (1975), and the less potential interference in recall. Thus, complex executive instructions should depress recall of the last few serially accessed items, and unrestrictive executive instructions should depress the total recall curve as compared with restrictive executive instructions.

The latency of responses should be indicative of search time through memory. Short latencies should be an indication of within-category or subcategory sampling, while longer latencies should reflect the search for new subcategories or rehearsal of recently produced items. Since individuals either recall a high proportion of the items of a category or fail to recall any of the items of a category (Cohen, 1966), the failure to recall items with long latencies during initial production would support the notion that such items represent the sampling of new categories or subcategories. This prediction is in direct opposition to the finding from experimenterpresented materials that the longer the latency period between items, the higher the probability of recall (Cooper \& Pantle, 1967). 


\section{METHOD}

\section{Subjects}

The participants in this study were 16 volunteer undergraduate students at Hunter College who were randomly assigned to one of four between-subjects treatments, producing 4 students per treatment. Five other students, distributed across the different treatments, were eliminated for failure to produce a sufficient number of responses for analysis.

\section{Procedure}

Each student sat in a small cubicle at a table across from the experimenter. The equipment on the table was a microphone, a stopwatch, a tape recorder, and the experimenter's instruction sheets. The student was told that this was a word association and memory study. One group of students was instructed to respond with whatever word that came to mind and to keep giving words (the free condition). A second group was told to respond with whatever word came to mind and to continue to give associates of the stimulus word (the standard condition). A third group of students was instructed to repeat the stimulus word after each response and, as in the standard condition, to continue to give associates of the stimulus word (the target-repeat condition). The fourth group was given a common category label and was instructed to respond with members of the category (the example condition).

There was one practice trial using as a stimulus the category label "fruit" for the example condition or the word "apple" for the other conditions. The experimenter terminated the procedure after the student had given 16 responses or after $30 \mathrm{sec}$ had elapsed from the previously given response, whichever came first. The students then were asked to recall the responses in any order that came to mind. It was explained that there would be eight test stimuli and that, after the subjects had responded to half the stimuli, the word "recall" would signal the memory test; on the other trials the signal "count" would be given, followed by a number from which the student was to count backward by units of three until the experimenter gave the "recall" cue. The number given was a randomly chosen two-digit number above 85 . Four different randomly presented numbers were used. The experimenter gave the "recall" cue after $30 \mathrm{sec}$ of counting. The four delay and four no-delay trials were randomly predetermined by the experimenter. The stimulus was both spoken by the experimenter and presented on a 4 x 6 in. index card.

The stimuli were the names of categories from the Battig and Montague (1969) norms in the example condition or the most typical associate within that category for the other conditions. The categories randomly chosen with the most typical associate in parentheses were a relative (aunt), a color (blue), a country (France), a sport (football), an article of clothing (shirt), a kind of money (dollars), a male's first name (John), and a college or university (Harvard). Harvard was the most common response after the names of the institutions in which the norms were established had been eliminated.

\section{RESULTS}

Initial responses were partitioned into quartiles, and the proportion of responses subsequently recalled for each quartile for each student was recorded for the four trials with no-delay task and the four trials with a delay task. A three-factor analysis of variance was performed on these data with repeated measures on the serial position and delay vs. no-delay task factors. The type of instruction resulted in statistically significant differences $[F(3,12)=3.50, p<.05]$. The example executive instruction produced the best recall: $87 \%$ of the initially produced responses. The standard and target-repeat executive instruction produced $84 \%$ and $75 \%$ recall, respectively, followed by the free executive instruction with $71 \%$ recall.

The serial position of the responses was significant $[F(3,36)=4.42, p<.01]$. First quartile retention was highest with $84 \%$, dropping to $79 \%$ and $74 \%$ in the second and third quartiles, respectively, and rising to $81 \%$ recall for the fourth quartile. Thus, the serial position effect appears to be evident averaged across all other conditions. Neither the presence or absence of a delay task nor any of the interactions approached significance.

The latency during response production was recorded to the nearest $1 \mathrm{sec}$; latencies of $3 \mathrm{sec}$ or less to the stimulus or a previous response were classed as short, and those taking $4 \mathrm{sec}$ or longer to produce were classed as long. For each quartile of the 16 responses produced, the proportion of the responses that was subsequently recalled and those responses not recalled with long latencies were calculated. A three-factor analysis of variance was performed with one factor as the types of executive instructions repeated measures on the serial position and latency factors. The latency factor was significant $[F(1,12)=14.62, p<.01]$. Only $26 \%$ of the responses recalled had long latencies, compared with $35 \%$ of the responses not recalled. The serial position of the responses also produced significant differences $[F(3,36)=9.72, p<.01]$. The proportion of responses with long latencies that was subsequently recalled increased through each successive quartile from $20 \%$ to $24 \%$ to $34 \%$ to $45 \%$ for the last quartile. None of the other effects was significant. The latency of response for the different executive instructions and the occurrence of a delay task was compared for the production and recall of responses. This three-factor analysis of variance produced a significant difference only between the latency of responding to the initial stimuli ( $3.8 \mathrm{sec} /$ response) and response latency during recall $(2.4 \mathrm{sec} /$ response $)[F(1,12)=20.72, p<.01]$.

There were two troublesome aspects of data collection: responses repeated during initial production and extralist responses in recall. Analyses of variance were performed for the incidences of these responses, but no differences were found to be due to type of executive instructions or the occurrence of delay tasks. There were, however, $4 \%$ repeats during recall, compared with only $2 \%$ during response production $[\mathrm{F}(1,12)=4.73$, $\mathrm{p}<.05$ ]. Of all responses given during recall, 5.3\% were extralist responses. There was no significant difference in recall as a function of the position of the task in the sequence of eight tasks.

\section{DISCUSSION}

Retrieval of information from storage was shown to be similar to encoding information into storage through the 
presence of a serial position effect in recall and also different, since long response latencies decreased the probability of recall. The presence of delay tasks did not affect behavior in this study. This lack of an effect that is commonly shown could be due to the absence of the effect when retrieving information from storage. However, design factors are likely to be responsible. The presence of repeated responses reduced the number of accessible responses to be recalled in some cases because the experimenter could not discount such responses as they were being produced. Students gave somewhat fewer than the requested 16 responses in 1.4 of the 8 categories, thus necessitating the use of fewer than 4 responses per quartile, which could affect the likelihood of a delay effect as it is based on only the last quartile. Finally, since students experienced delay instructions on some but not all occasions, they might have responded to each stimulus as if a delay task were forthcoming. It would be better in future studies not to use repeated measures on this factor.

The failure of items with long latencies, especially those produced early, to be subsequently recalled is similar to the findings of Cohen (1966) for category recall, and it does give support to the notion that long pauses indicate searching within memory storage. A within-subjects measure of such pauses might be a better indicator of search time than the cross-subjects measure of $4 \mathrm{sec}$ used with these data. The difficulty that students had in producing 16 responses to the stimuli used in this study and the relatively long latencies in producing the responses that were obtained were surprising. The high proportion of items produced, and produced more quickly, during recall contrasts sharply with the initial production of responses from memory storage. Certainly, in a recognition task of category membership, accuracy would be higher and latencies would be much shorter. The presence of proactive interference (although there was no evidence of such interference building up across tasks), rehearsal for subsequent recall, or limitations upon search mechanisms might be responsible. Some students might find it difficult to persist in searching within one category without the opportunity to scan across other categories (Zippel, 1975).

The free association executive instruction produced the poorest recall compared with the target-repeat and standard instructions, which all used the same stimuli, and the example instruction, which used category labels as stimuli. It appears that recall decreases as the size of the available pool of items delimited by the instruction increases. The example instruction, although it uses a different cuing stimulus, refers to the limited set of category members as responses, whereas the standard and target-repeat instructions more broadly include any associate of the stimulus and the free instruction permits any verbal response. The target-repeat and standard instructions both refer to the same available population of responses. The target-repeat instruction was more complex to apply, and it produced poorer recall than the standard instruction. Thus, the data indicate that complexity and size of available response population may be separable factors that influence recall. From these data there is only the most tentative evidence that instructions may have some representation in STM storage.

\section{REFERENCES}

Anderson, J. R., \& Bowe R, G. H. Human associative memory. Washington, D.C: Winston, 1973.

Battig, W. F., \& Montague, W. E. Category norms for verbal items in 56 categories: A replication and extension of the Connecticut category norms. Journal of Experimental Psychology Monograph, 1969, 80(3, Pt. 2).

Conen, B. H. Some or none characteristics of coding. Journal of Verbal Learning and Verbal Behavior, 1966, 5, 182-187.

Cooper, E. H., \& Pantle, A. J. The total-time hypothesis in verbal learning. Psychological Bulletin, 1967, 68, 221-234.

Glanze R, M. Storage mechanisms in recall. In G. H. Bower (Ed.), The psychology of learning and motivation (Vol. 5). New York: Academic Press, 1972.

Johnson, N. F. The role of chunking and organization in the process of recall. In G. H. Bower (Ed.), The psychology of learning and motivation (Vol. 4). New York: Academic Press, 1970.

Kegle, S. W. Attention and human performance. Pacific Palisades, Calif: Goodyear, 1973.

Mandle R, G. Organization and memory. In K. Spence \& J. Spence (Eds.), The psychology of learning and motivation (Vol. 1). New York: Academic Press, 1967.

MEYER, D. E. Long-term memory retrieval during the comprehension of affirmative and negative sentences. In R. A. Kennedy \& A. L. Wilkes (Eds.), Studies in long-term memory. London: Wiley, 1975.

Murdock, B. B., JR. Human memory: Theory and data. Hillsdale, N.J: Erlbaum, 1974.

Posner, M. I., Rossman, E. Effect of size and location of informational transforms upon short-term retention. Journal of Experimental Psychology, 1965, 70, 496-505.

Wickelgren, W. A. Size of rehearsal group and short-term memory. Journal of Experimental Psychology, 1964, 68, 413419.

ZIPPEL, B. Information retrieval from long-term memory. Journal of General Psychology, 1975, 93, 73-85.

(Received for publication October 24, 1979.) 